\title{
DISTRIBUTED STATE ESTIMATION FOR HIDDEN MARKOV MODELS WITH DYNAMIC QUANTIZATION AND RATE ALLOCATION ${ }^{1}$
}

\author{
Minyi Huang*, Subhrakanti Dey* \\ * Dept. Electrical and Electronic Engineering, University of \\ Melbourne, Parkville, 3010 Victoria, Australia.
}

\begin{abstract}
This paper considers state estimation of hidden Markov models by sensor networks. By employing feedback from the fusion center to the sensor nodes, a dynamic quantization scheme is proposed and analyzed by a stochastic control approach. Dynamic rate allocation is also considered. Copyright (c)2005 IFAC
\end{abstract}

Keywords: Hidden Markov models, sensor networks, dynamic quantization.

\section{INTRODUCTION}

Sensor networks have gained intensive research interest due to their wide range of current and potential applications in environment surveillance, detection and estimation, etc. (Chong and $\mathrm{Ku}-$ mar, 2003). In such networks, sensors are geographically distributed for sending data to a fusion center (FC). Due to their limited computational capacity and scarce communication rate shared by all sensors to communicate with the fusion center, it is impossible for the sensors to send their exact measurements and instead, only a quantized output is transmitted. Then the fusion center needs to combine the data received from all sensors to make a decision or form an estimate.

Within the context of statistical signal processing, an important application of sensor networks is state estimation of random processes, since in reality sensor networks operate in a time-varying environment and the resulting measurements are governed by certain dynamic models (Fletcher et al., 2004). For describing certain applications of interest, the underlying random process may be taken as a Markov chain and analyzed by the hidden Markov chain techniques (Shue et al., 2001).

1 This work was partially supported by ARC.
Also, see (Krishnamurthy, 2002) for multiple sensor scheduling for hidden Markov models. In general the quantization optimization problem for sensor networks is not a trivial task even when the Markov chain has only very few states. This may be attributed to the high complexity in the associated nonconvex optimization problems.

This paper considers the estimation of finite state Markov chains by sensor networks. For computational tractability, binary quantization is employed at the sensors. In general, such a quantization scheme can only transmit very coarse information, and traditionally the network performance is improved by increasing the number of sensors. There has been an extensive literature on binary sensors in the context of hypothesis testing; see (Chamberland and Veeravalli, 2003) and references therein.

Instead of improving the estimation by increasing the number of sensors, the present work will adopt another approach by establishing feedback from the fusion center to the sensors so that a certain coordination of the sensors may be maintained. The consequence of the feedback is that the usual static quantization scheme is then replaced by a dynamic one. Evidently, in this paper the communication pattern between the fusion center and the 
sensors is more complicated compared to unidirectional sensor networks. However, this approach has the potential to reduce the network complexity from another point of view, i.e, in order to achieve a prescribed performance, one only needs to implement fewer sensor nodes compared to the case without feedback. This kind of feedback information pattern has been employed for performance improvement in the sensor networks literature, but mainly in the context of hypothesis testing (Pados et al., 1995; Alhakeem and Varshney, 1996), and is referred to as decision feedback.

The paper is organized as follows: Section 2 formulates the state estimation problem and in Section 3 , an equivalent stochastic control problem is formulated. The dynamic programming equation is studied in Section 4. Section 5 presents numerical results. In Section 6 rate allocation is analyzed. Section 7 concludes the paper.

\section{SYSTEM MODEL}

Let $\left\{X_{t}, t \geq 1\right\}$ be a discrete time Markov chain with state space $S=\left\{s_{1}, \cdots, s_{n}\right\}$ and transition matrix $P=\left[p_{i j}\right]$, where $p_{i j}=P\left(X_{t+1}=s_{j} \mid X_{t}=\right.$ $\left.s_{i}\right)$. Assume without loss of generality that $s_{1}<$ $\cdots<s_{n}$. Let the measurement of the $M$ sensors be specified by

$$
Y_{m, t}=X_{t}+W_{m, t} \quad 1 \leq m \leq M .
$$

A similar model for a two state Markov chain with one sensor has been studied in (Shue et al., 2001) and performance analysis is based upon static quantization with different quantization levels. Write (2.1) in the vector form

$$
Y_{t}=A X_{t}+W_{t}
$$

where $Y_{t}=\left[Y_{1, t}, \cdots, Y_{M, t}\right]^{T}, A=[1, \cdots, 1]^{T}$ and $W_{t}=\left[W_{1, t}, \cdots, W_{M, t}\right]^{T}$. For simplicity, the noise $\left\{W_{t}\right\}$ is assumed to be a sequence of i.i.d. vector random variables.

For a set of $M$ binary sensors, any given quantization scheme is specified by $M$ sequences of constants $\left\{r_{m, t}, t \geq 1\right\}, 1 \leq m \leq M$, where $r_{m, t}$ is used to partition the range space of $Y_{m, t}$. Let $r_{t}=\left(r_{1, t}, \cdots, r_{M, t}\right)$, and write the quantization sequence $\left\{r_{t}, t \geq 1\right\}=\left\{\left(r_{1, t}, \cdots, r_{M, t}\right), t \geq 1\right\}$. At time $t$, let the data (to be called message) that the fusion center receives from the $m$-th sensor be denoted by $Y_{m, t}^{q}$. One may take any two distinct alphabets $a_{1}$ and $a_{2}$ such that the events $\left\{Y_{m, t}<r_{m, t}\right\}$ and $\left\{Y_{m, t} \geq r_{m, t}\right\}$ are equivalent to $\left\{Y_{m, t}^{q}=a_{1}\right\}$ and $\left\{Y_{m, t}^{q}=a_{2}\right\}$, respectively. Hence the received message at the fusion center is

$$
Y_{m, t}^{q}= \begin{cases}a_{1} & Y_{m, t}<r_{m, t} \\ a_{2} & Y_{m, t} \geq r_{m, t}\end{cases}
$$

Let $Y_{t}^{q}=\left[Y_{1, t}^{q}, \cdots, Y_{m, t}^{q}\right]^{T}$ and denote $Y_{t}^{q}$ by

$$
Y_{t}^{q}=\mathcal{Q}\left(r_{t}, Y_{1, t}, \cdots, Y_{M, t}\right),
$$

where the map $\mathcal{Q}: \mathbb{R}^{M} \times \mathbb{R}^{M} \rightarrow\left\{a_{1}, a_{2}\right\}^{M}$ is determined from (2.3) in an obvious manner.

For each sequence $\left\{r_{t}\right\}$, the long-term mean squared error for the state estimation is given as

$$
J(r)=\limsup _{N \rightarrow \infty} \frac{1}{N} \sum_{t=1}^{N} E\left|X_{t}-\widehat{X}_{t}\right|^{2}
$$

where the sequence $\left\{r_{t}, t \geq 1\right\}$ is simply indicated as $r$ and the estimate $\widehat{X}_{t}$ is a Borel measurable function of the sequence $\left\{Y_{k}^{q}, k \leq t\right\}$. In this paper, $|x| \triangleq \sum_{i=1}^{n}\left|x_{i}\right|$ for $x \in \mathbb{R}^{n}$.

\section{THE EQUIVALENT OPTIMAL CONTROL PROBLEM}

The dynamic quantization problem may be regarded as a generalized stochastic control problem in which $\left\{r_{t}\right\}$ affects the observation $Y_{t}^{q}$ at the fusion center, but the state variable $\left\{X_{t}\right\}$ is autonomous. Since the fusion center is generally equipped with a high computational and data storage capacity, the parameters $r_{t}$ are computed at the fusion center as a function of $\left(Y_{1}^{q}, \cdots, Y_{t-1}^{q}\right)$. In other words, $r_{t}$ is adapted to $\mathcal{F}_{t-1} \triangleq \mathcal{F}\left(Y_{i}^{q}, i \leq t-1\right)$ which is the $\sigma$-algebra generated by the past observations. In further analysis, a recursively calculated sufficient statistic shall be identified such that $r_{t}$ need not be determined using the overall history $\left(Y_{1}^{q}, \cdots, Y_{t-1}^{q}\right)$ when the sufficient statistic is computed at each step. Once $r_{t}$ is computed, the entry $r_{m, t}$ is sent from the fusion center to the $m$-th sensor. In this framework, the distributed nature of the network is preserved in the sense that the data is preprocessed at the sensor node level based upon which the fusion center forms a final estimate, and no direct communication exists between the sensors except that each sensor receives feedback commands from the fusion center.

Define the so-called information state (Kumar and Varaiya, 1986) $\theta_{t}=\left[\theta_{1, t}, \cdots, \theta_{n, t}\right]^{T}$, where

$$
\theta_{i, t}=E\left[X_{t}=s_{i} \mid \mathcal{F}_{t}\right], \quad 1 \leq i \leq n, \quad t \geq 1 .
$$

By the Bayesian rule, $\theta_{t}$ is recursively given as

$$
\begin{aligned}
\theta_{t+1} & =\frac{1}{z_{t+1}} Q\left(s_{1}, \cdots, s_{n}, r_{t+1}, Y_{t+1}^{q}\right) P^{T} \theta_{t} \\
& \triangleq \frac{1}{z_{k+1}} T\left(s_{1}, \cdots, s_{n}, r_{t+1}, Y_{t+1}^{q}\right) \theta_{t}
\end{aligned}
$$

where $P$ is the transition matrix of $\left\{X_{t}\right\}, z_{t+1}$ is a normalizing factor such that $\left|\theta_{t+1}\right|=1$, and

$$
\begin{aligned}
& Q\left(s_{1}, \cdots, s_{n}, r_{t}, y_{t}^{q}\right) \\
= & \operatorname{Diag}\left[F\left(s_{1}, r_{t}, y_{t}^{q}\right), \cdots, F\left(s_{n}, r_{t}, y_{t}^{q}\right)\right]_{n \times n},
\end{aligned}
$$


where $y_{t}^{q}$ denotes a value for $Y_{t}^{q}$. The matrix $T\left(s_{1}, \cdots, s_{n}, r_{t}, y_{t}^{q}\right)$ may be simply written as $T\left(r_{t}, y_{t}^{q}\right) . F\left(s_{i}, r_{t},\left(a_{i_{1}}, \cdots, a_{i_{M}}\right)\right)=\int_{\mathcal{A}\left(r_{t}\right)} f\left(y_{1}-\right.$ $\left.s_{i}, \cdots, y_{M}-s_{i}\right) d y_{1} \cdots d y_{M}$ with $\mathcal{A}\left(r_{t}\right) \triangleq\{y \in$ $\left.\mathbb{R}^{M}, \mathcal{Q}\left(r_{t}, y\right)=\left(a_{i_{1}}, \cdots, a_{i_{M}}\right)\right\}$, where $f$ is the joint probability density for $W=\left(W_{1, t}, \cdots, W_{M, t}\right)^{T}$ and $\mathcal{Q}$ is defined in (2.4). In the special case of two sensors, i.e., $M=2$, then $F\left(s_{i}, r,\left(a_{1}, a_{1}\right)\right)=$ $\int_{-\infty}^{r_{1}} \int_{-\infty}^{r_{2}} f\left(y_{1}-s_{i}, y_{2}-s_{i}\right) d y_{1} d y_{2}$ etc., where $\left(a_{i}, a_{j}\right)$ corresponds to an outcome of $Y_{t}^{q}$ and determines a specific integration region.

Given $\mathcal{F}_{t}$, the conditional expectation of $X_{t}$ is

$$
\widehat{X}_{t}=E\left[X_{t} \mid \mathcal{F}_{t}\right]=\sum_{i=1}^{n} s_{i} \theta_{i, t} .
$$

In fact, for any given quantization sequence $\left\{r_{t}\right\}$, $E\left|X_{t}-\widehat{X}_{t}\right|^{2}=\inf E\left|X_{t}-Z_{t}\right|^{2}$, where $Z_{t}$ is any random variable adapted to $\mathcal{F}_{t}$. By virtue of this fact, in future analysis $\widehat{X}_{t}$ in the cost (2.5) is always taken as the conditional expectation (3.2). Set the conditional cost

$c\left(\theta_{t}\right)=E\left[\left|X_{t}-\widehat{X}_{t}\right|^{2} \mid \mathcal{F}_{t}\right]=\sum_{i=1}^{n}\left[s_{i}-\sum_{j=1}^{n} s_{j} \theta_{j, t}\right]^{2} \theta_{i, t}$,

which is computed by (3.2). In the special case of $n=2,\left.c\left(\theta_{t}\right)\right|_{n=2}=\left(s_{1}-s_{2}\right)^{2} \theta_{1, t} \theta_{2, t}$.

Now the optimal estimation problem associated with (2.5) may be equivalently expressed as

$(\mathbb{P})$ minimize $J(r, \theta)=\limsup _{N \rightarrow \infty} \frac{1}{N} \sum_{t=1}^{N} E\left[c\left(\theta_{t}\right) \mid \theta_{1}=\theta\right]$,

for which $r_{t}$ is adapted to $\mathcal{F}_{t-1}$. Notice that the fusion center cannot directly minimize the cost (2.5) since it has no exact knowledge on $X_{t}$. However, it can solve the problem $(\mathbb{P})$ since $\theta_{t}$ may be recursively computed using $Y_{i}^{q}, i \leq t$. Indeed, $(\mathbb{P})$ is a standard stochastic control problem with complete information, and its associated dynamic programming (Bellman) equation is given as

$$
\begin{aligned}
\lambda+h(\theta) & =\min _{r}\left[c(\theta)+\sum_{Y^{q}}\left|T\left(r, Y^{q}\right) \theta\right| h\left(\frac{T\left(r, Y^{q}\right) \theta}{\left|T\left(r, Y^{q}\right) \theta\right|}\right)\right] \\
& \triangleq \min _{r} \Phi(\theta, r)
\end{aligned}
$$

where $Y^{q} \in\left\{a_{1}, a_{2}\right\}^{M} . h(\theta)$ is called the differential cost. Let $S_{1} \triangleq\left\{\alpha \in \mathbb{R}_{+}^{n}, \sum_{i=1}^{n} \alpha_{i}=1\right\}$.

Theorem 1. Assume there exist $\lambda \in \mathbb{R}$ and a bounded function $h: S_{1} \rightarrow \mathbb{R}$, satisfying (3.4), and there is a measurable function $r=g(\theta)$ such that $g(\theta)=\arg \min _{r} \Phi(\theta, r)$. Then the quantization with $r_{t}=g\left(\theta_{t-1}\right)$ minimizes the cost in (3.3) with the optimal cost $\lambda$.

Remark: The theorem is essentially an adaptation of the standard verification theorem for optimal
Markov decision problems with Borel state spaces. $r_{1}$ may be set as any fixed value. Existence of a solution to equation (3.4) is insured based upon mild conditions in terms of its exponentially discounted version; see e.g. (Fernandez-Gaucherand et al., 1991).

For static quantization, i.e., all $r_{t}=r$, the resulting cost $\lambda_{0}$ may be specified as follows:

$\lambda_{0}+h_{0}(\theta)=c(\theta)+\sum_{Y^{q}}\left|T\left(r, Y^{q}\right) \theta\right| h_{0}\left(\frac{T\left(r, Y^{q}\right) \theta}{\left|T\left(r, Y^{q}\right) \theta\right|}\right)$

with $r=\left(r_{1}, \cdots, r_{M}\right)$, which is of a degenerate form of (3.4) since the domain for $r$ is now a singleton. (3.5) is useful for the performance calculation of any static binary quantizer.

\section{DISCRETIZATION OF THE BELLMAN EQUATION}

From a numerical computational point of view, a solution to (3.4), if existing, is hard to solve since for a fixed $\theta$, the right hand side of (3.4) is a nonconvex function of the variable $r \in \mathbb{R}^{M}$. For numerical tractability, in this section a variant of the problem $(\mathbb{P})$ is considered where $r$ is restricted to a finite set. The following steps are carried out:

(a) Choose a finite set as the range space of $r$;

(b) As a suboptimal approximation to $\mathbb{P}$, discretize the information state and derive a finite dimensional equation which, in fact, corresponds to a well defined optimal Markov decision problem with finite states.

(c) Solve the fully discretized Bellman equation by the relative value iteration algorithm.

For notational and computational simplicity, the same finite subset of $\mathbb{R}$ is employed for optimizing each entry $r_{m}$ in $r \in \mathbb{R}^{M}, 1 \leq m \leq M$. Now, let the range space of $r_{m, t}$ be $L_{d}=\left\{\gamma_{1}, \cdots, \gamma_{d}\right\} \subset \mathbb{R}$. Hence $r$ shall be chosen from the set $L_{d}^{M}$. Write the corresponding Bellman equation as

$\lambda+h(\theta)=\min _{r_{m} \in L_{d}}\left[c(\theta)+\sum_{Y^{q}}\left|T\left(r, Y^{q}\right) \theta\right| h\left(\frac{T\left(r, Y^{q}\right) \theta}{\left|T\left(r, Y^{q}\right) \theta\right|}\right)\right]$

Let us introduce the assumption:

(H1) For any $r \in L_{d}^{M}$ and $y^{q} \in\left\{a_{1}, a_{2}\right\}^{M}$, the matrix $T\left(r, y^{q}\right)$ is strictly positive.

Notice that (H1) holds under very mild conditions. For instance, it holds for non-degenerate Gaussian noise and positive $P$.

Proposition 1. Under (H1), there exist $\lambda$ and a bounded function $h$ satisfying the equation (4.1).

The proof is obtained by considering the exponentially discounted version of the cost as in 


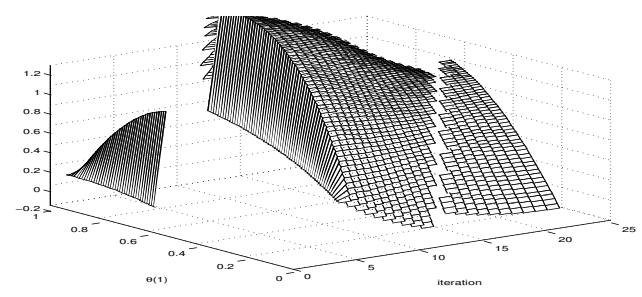

Fig. 1. Iteration for $h$; each slice corresponds to the curve of $h$ at a fixed iterate.

(Fernandez-Gaucherand et al., 1991), and (H1) may be relaxed such that $T\left(r, Y^{q}\right)$ is only primitive. The details are omitted here.

For notational simplicity, the numerical procedure for solving (4.1) is described with $n=2$, i.e., $\theta \in \mathbb{R}^{2}$. The same procedure can be employed for the case $n>2$. For $n=2$, let the range space $S_{1}$ of $\theta$ be discretized with a step size $\frac{1}{N}$. Let $S_{1, N}=\left\{\left[\frac{k}{N}, 1-\frac{k}{N}\right]^{T}, k=0, \cdots, N\right\}$. Take $\theta \in$ $S_{1, N}$ for the left hand side of (4.1). However, due to the linear transform and normalization inside $h$, the right hand side of (4.1) involves values of $h$ at points outside $S_{1, N}$. Hence this cannot induce an equation only in terms of values of $h$ on the grid $S_{1, N}$. To overcome this difficulty, consider an approximation by rounding off $\theta^{\prime}=\frac{T \theta}{|T \theta|}$ to the closest point $\theta^{\prime \prime}$ in $S_{1, N}$, and replacing $h\left(\theta^{\prime}\right)$ by $h\left(\theta^{\prime \prime}\right)$. This procedure leads to a fully discretized equation:

$\lambda+h\left(l_{k}\right)=$

$\min _{r_{i} \in L_{d}}\left[c\left(l_{k}\right)+\sum_{Y^{q}}\left|T\left(r, Y^{q}\right) l_{k}\right| h\left(\left[\frac{T\left(r, Y^{q}\right) l_{k}}{\left|T\left(r, Y^{q}\right) l_{k}\right|}\right]_{\text {round }}\right)\right]$

where $l_{k} \in S_{1, N}$, and for $\theta=\left[\beta_{1}, \beta_{2}\right]^{T} \in S_{1}$, $[\beta]_{\text {round }}=\left(\left[\beta_{1}\right]_{\text {round }}, 1-\left[\beta_{1}\right]_{\text {round }}\right)^{T}$ with

$\left[\beta_{1}\right]_{\text {round }}=\left\{\begin{array}{cl}\frac{k}{N} & \text { for } \quad \beta \in\left(\frac{k}{N}-\frac{1}{2 N}, \frac{k}{N}+\frac{1}{2 N}\right] \\ 0 & \text { for } \beta \in\left[0, \frac{1}{2 N}\right] \\ 1 & \text { for } \quad \beta \in\left(1-\frac{1}{2 N}, 1\right] .\end{array}\right.$

Notice that for a fixed $l_{k} \in S_{1, N}$ the summation on the right hand side of (4.2) involves the value of $h$ at four point $l_{k}^{(i)}$ (derived from the rounding off procedure), each associated with a weight coefficient $\delta_{k}^{(i)}$, depending on $Y^{q}$ and satisfying $\sum_{i} \delta_{k}^{(i)}=1$. Hence this gives the Bellman equation for a standard finite state Markov decision problem and then (4.2) can be solved by the relative value iteration method which converges to its exact solution; see (Bertsekas, 1995).

\section{NUMERICAL EXPERIMENTS}

5.1 Estimating a two state Markov chain via two sensors

Since $\theta(1)+\theta(2)=1$, the differential cost $h$ is parametrized in terms of $\theta(1)$ and denoted as

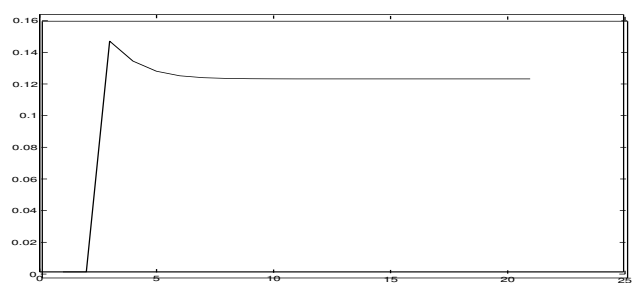

(a)

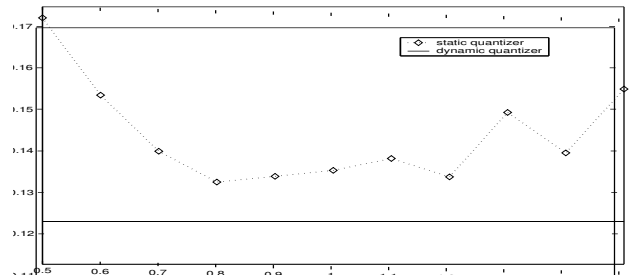

(b)

Fig. 2. (a) - convergence of the cost during iteration to 0.11996; (b) - the lowest cost attained by static quantization in $L_{d}^{\prime}$ is 0.129144 with $r=0.8$ for two sensors.

$h(\theta(1))$. The transition matrix for $\left\{X_{t}\right\}$ is $P=$ $\left[\begin{array}{ll}0.8 & 0.2 \\ 0.4 & 0.6\end{array}\right]$ and the two noise components are independent and Gaussian with $\sigma_{1}^{2}=\sigma_{2}^{2}=0.5$. $s_{1}=0$ and $s_{2}=2$. The step size $\frac{1}{N}=0.01$ is used for discretization of $\theta$. The set $L_{d}=$ $\{0,0.1,0.2, \cdots, 1.9,2.0\}$ is used in (4.2). The pair $(\lambda, h)$ is computed using the relative value iteration algorithm by 20 iterates. Fig. 1 shows the convergence of the differential cost. The optimal cost $(\lambda)$ converges to 0.11996 as shown in Fig. $2(\mathrm{a})$.

The cost for static quantizers is computed where the two sensors' quantization is specified by a common scalar parameter $r \in L_{d}^{\prime}=\{0.5,0.6, \cdots, 1.5\}$ and the associated costs for different $r$ are plotted in Fig. 2(b). The solid line gives the optimal cost 0.11996 for the dynamic quantization.

\subsection{Tracking multiple state slow Markov chains} via a single sensor

In the example only one sensor is employed for estimating a slow Markov chain with measurement $Y_{t}=X_{t}+W_{t}$. With the quantization parameter $r_{t} \in \mathbb{R}$, the output is: $Y_{t}^{q}=a_{1}$ if $Y_{t}<r_{t}$, and $Y_{t}^{q}=a_{2}$ if $Y_{t} \geq r_{t}$. Here the i.i.d. Gaussian noise has variance $\sigma^{2}$ and $\left\{X_{t}\right\}$ has states $\left\{s_{1}=0, s_{2}=\right.$ $\left.1, s_{3}=2.5\right\}$ and transition matrix

$$
P=\left[\begin{array}{ccc}
0.9 & 0.1 & 0 \\
0.1 & 0.8 & 0.1 \\
0 & 0.15 & 0.85
\end{array}\right]
$$

See Table 1 for the optimal cost for dynamic quantization.

For the static quantizers with $r$ chosen from $\{0.5,0.6, \cdots, 2.0\}$ (with step size 0.1), the lowest 
Table 1. Costs computed by 25 iterates

\begin{tabular}{|c|c|c|}
\hline$\sigma^{2}$ & static quantizer & dynamic quantizer \\
\hline 0.5 & $0.2779(r=1.5)$ & 0.259 \\
\hline 0.3 & $0.2287(r=1.5)$ & 0.1932 \\
\hline 0.1 & $0.1666(r=1.7)$ & 0.0814 \\
\hline
\end{tabular}

attainable cost is listed in Tables 1 with the associated value for $r$. It is shown when the noise variance decreases, the relative improvement in performance by dynamic quantization increases.

\section{MODE DEPENDENT OBSERVATION AND DYNAMIC RATE ALLOCATION}

In this section, let the observation of the sensor nodes be given by

$$
Y_{m, t}=g_{m}\left(X_{t}, Z_{t}\right)+W_{m, t} \quad 1 \leq m \leq M,
$$

which may be written in the vector form:

$$
Y_{t}=G\left(X_{t}, Z_{t}\right)+W_{t} .
$$

Here $X_{t}$ is the Markov chain with state space $S=$ $\left\{s_{1}, \cdots, s_{n}\right\}$, and $Z_{t}$ will be specified later. (6.1) shall be termed mode dependent observations. Some motivational interpretation for this model is in order.

(a) Location dependent measurements Consider an object having random visits between multiple regions $R_{i}$, each corresponding to a sensor. The sensor measurements reflect both the spatial position $X_{t}$ of the object (specified up to region) and its randomly varying motion parameter $Z_{t}$ (e.g., altitude, velocity, angle, etc., or their combination).

(b) Action dependent measurements for maneuvering targets - In the literature (Mazor et al., 1998), a typical modelling of a single maneuvering target is to employ a stochastic hybrid dynamical system, where a finite state Markov chain $X_{t}$ describes the maneuvering actions which drive the evolution of the target state $Z_{t}$. Assume multiple sensors are employed for target tracking such that each sensor is particularly suitable (e.g., giving a higher measurement gain) for dealing with a specific maneuvering action.

Let (6.1) be further simplified as:

$$
Y_{m, t}=g_{m}\left(X_{t}\right) Z_{t}+W_{m, t}, \quad 1 \leq m \leq M,
$$

where $P\left(Z_{t+1}=s_{j}^{z} \mid Z_{t}=s_{i}^{z}, X_{t}=s_{k}\right)=p_{i j, k}^{z}$, for which $Z_{t}$ has state space $S^{z}=\left\{s_{1}^{z}, \cdots, s_{\bar{n}}^{z}\right\}$. The i.i.d. noise $W_{t}$ has a probability density $f$. Indeed, the above modelling of $Z_{t}$ may be regarded as a simplified discrete approximation of the hybrid continuum modelling of the target state in the tracking literature; see (Mazor et al., 1998). Here $X_{t}$ models the multiple modes.

Within this modelling paradigm it is of interest to consider dynamic rate allocation under the condition that the total rate of the sensors is constrained due to the shared communication channel. The intuitive justification of dynamic rate allocation with the mode dependent observations is that, if it is inferred from posterior information that the system is more likely to be operating in mode $s_{m_{i}}$ for which sensor $S_{i}$ has a higher measurement gain, then this sensor should be assigned more rate for refined estimates, and that the consequently reduced rate for other sensors should result in far less performance loss since their observations are less useful due to their low signal to interference ratio.

The main idea for dynamic rate allocation is that one may choose the quantization parameters $r_{t}$ such that the corresponding partition at the sensors does not produce a total rate (or total number of quantization levels) exceeding a specified number, and it is allowed to split the number of quantization levels unevenly at the sensors.

For notational simplicity, in the following a system of two sensors is analyzed where each of $X_{t}$ and $Z_{t}$ has two states, i.e., $S=\left\{s_{1}, s_{2}\right\}$ and $S^{z}=$ $\left\{s_{1}^{z}, s_{2}^{z}\right\}$. The generalization to the case of more states is evident. Denote the transition matrix of $X_{t}$ by $P=\left(p_{i j}\right)_{1 \leq i, j \leq 2}$, and let the transition matrix of $Z_{t}$ given $\bar{X}_{t}=s_{i}$ be given as $\left.P^{z}\right|_{X_{t}=s_{1}}=$ $\left(p_{i j}^{z}\right)_{1 \leq i, j \leq 2},\left.P^{z}\right|_{X_{t}=s_{2}}=\left(\hat{p}_{i j}^{z}\right)_{1 \leq i, j \leq 2}$.

The quantization scheme consists of one binary sensor with alphabet set $\left\{a_{1}, a_{2}\right\}$, and a ternary one with alphabet set $\left\{a_{1}, a_{2}, a_{3}\right\}$. Hence the total number of quantization levels is 5 . Furthermore, let the parameter $r_{b i n}$ for the binary sensor be chosen from the set $L_{d_{1}}^{b}=\left\{\gamma_{1}, \cdots, \gamma_{d_{1}}\right\}$. The ternary quantizer is specified by a pair $r_{\text {ter }}$ in the set $L_{d_{2}}^{t}=\left\{\left(\gamma_{1}^{i}, \gamma_{2}^{i}\right), 1 \leq i \leq d_{2}\right\}$. Now any quantizer, denoted simply as $r_{t}$, at time $t$ may be represented as $\left(\gamma_{1} ; \gamma_{2}, \gamma_{3}\right)$ with the first sensor being binary, or $\left(\gamma_{1}, \gamma_{2} ; \gamma_{3}\right)$ with the second being binary. Hence an admissible quantizer $r_{t}$ is an entry in the union $\left(L_{d_{1}}^{b} \times L_{d_{2}}^{t}\right) \cup\left(L_{d_{2}}^{t} \times L_{d_{1}}^{b}\right)$ of two sets, each being an ordered cartesian product. For instance, $\left(\gamma_{1}, \gamma_{2} ; \gamma_{3}\right)$ is in $L_{d_{2}}^{t} \times L_{d_{1}}^{b}$ where $\left(\gamma_{1}, \gamma_{2}\right) \in L_{d_{2}}^{t}$ and $\gamma_{3} \in L_{d_{1}}^{b}$. Once $r_{t}$ is selected, the message $Y_{t}^{q}=\left[Y_{1, t}^{q}, Y_{2, t}^{q}\right]^{T}$ received by the fusion center is an entry in $\left(\left\{a_{1}, a_{2}\right\} \times\left\{a_{1}, a_{2}, a_{3}\right\}\right) \cup$ $\left(\left\{a_{1}, a_{2}, a_{3}\right\} \times\left\{a_{1}, a_{2}\right\}\right)$. As in Section 2, denote the quantizer output by $Y_{t}^{q}=\mathcal{Q}\left(r_{t}, Y_{t}\right)$.

For the estimation of $\left(X_{t}, Z_{t}\right)$, the cost is specified by the weighted mean square error:

$$
J(r)=\limsup _{N \rightarrow \infty} \frac{1}{N} \sum_{t=1}^{N}\left[E\left|X_{t}-\widehat{X}_{t}\right|^{2}+\beta E\left|Z_{t}-\widehat{Z}_{t}\right|^{2}\right]
$$

where $\beta>0$. Define the information state $\theta_{t}=$ $\left[I_{11}, I_{12}, I_{21}, I_{22}\right]^{T}$ where $I_{i j}(t)=E\left[X_{t}=s_{i}, Z_{t}=\right.$ $\left.s_{j}^{z} \mid Y_{1}^{q}, \cdots, Y_{t}^{q}, r_{1}, \cdots, r_{t}\right]$. Here $Y_{t}^{q}$ denotes the quantized output of the sensors. Define 


$$
D=\left(\begin{array}{llll}
p_{11} p_{11}^{z} & p_{11} p_{12}^{z} & p_{12} p_{11}^{z} & p_{12} p_{12}^{z} \\
p_{11} p_{21}^{z} & p_{11} p_{22}^{z} & p_{12} p_{21}^{z} & p_{12} p_{22}^{z} \\
p_{21} \hat{p}_{11}^{z} & p_{21} \hat{p}_{12}^{z} & p_{22} \hat{p}_{11}^{z} & p_{22} \hat{p}_{12}^{z} \\
p_{21} \hat{p}_{21}^{z} & p_{21} \hat{p}_{22}^{z} & p_{22} \hat{p}_{21}^{z} & p_{22} \hat{p}_{22}^{z}
\end{array}\right)
$$

which is the transition probability matrix of the joint Markov process $\left(X_{t}, Z_{t}\right)$. Let

$$
Q\left(r_{t}, Y_{t}^{q}\right)=\operatorname{Diag}\left[Q_{11}, Q_{12}, Q_{21}, Q_{22}\right]\left(r_{t}, Y_{t}^{q}\right),
$$

where $Q_{i j}\left(r_{t}, Y_{t}^{q}\right)=\int_{\mathcal{Q}^{-1}\left(r_{t}, Y_{t}^{q}\right)} f\left(y_{1}-g_{1}\left(s_{i}\right) s_{j}^{z}, y_{2}-\right.$ $\left.g_{2}\left(s_{i}\right) s_{j}^{z}\right) d y_{1} d y_{2}$, and $\mathcal{Q}^{-1}\left(r_{t}, Y_{t}^{q}\right)=\left\{\left(y_{1}, y_{2}\right):\right.$ $\left.\mathcal{Q}\left(r_{t}, y_{1}, y_{2}\right)=Y_{t}^{q}\right\}$. The recursion for the information state is $\theta_{t+1}=\frac{1}{z_{t+1}} Q\left(r_{t+1}, Y_{t+1}^{q}\right) D^{T} \theta_{t} \triangleq$ $\frac{1}{z_{t+1}} T\left(r_{t+1}, Y_{t+1}^{q}\right) \theta_{t}$. The conditional cost is

$$
\begin{aligned}
c\left(\theta_{t}\right)= & \left(s_{1}-s_{2}\right)^{2}[\theta(1)+\theta(2)][\theta(3)+\theta(4)] \\
& +\beta\left(s_{1}^{z}-s_{2}^{z}\right)^{2}[\theta(1)+\theta(3)][\theta(2)+\theta(4)] .
\end{aligned}
$$

As in Sections 3-4, the Bellman equation may be written and then discretized. For reasons of space, the details are omitted here.

\subsection{Numerical simulation}

In the simulation, the system is specified as follows: $S=S_{z}=\{1,2\}$ and $g_{1}(1)=1, g_{2}(2)=$ $1.1, g_{1}(2)=g_{2}(1)=0.25, \beta=2$. The transition matrices are $P=\left[\begin{array}{cc}0.85 & 0.15 \\ 0.1 & 0.9\end{array}\right],\left.P^{z}\right|_{X_{t}=1}=$ $\left[\begin{array}{ll}0.6 & 0.4 \\ 0.3 & 0.7\end{array}\right],\left.P^{z}\right|_{X_{t}=2}=\left[\begin{array}{ll}0.8 & 0.2 \\ 0.1 & 0.9\end{array}\right]$. Let $L_{d_{1}}^{b}=$ $\{0.1,0.2,0.3,0.4\}$ and $L_{d_{2}}^{t}=\left\{\left(\gamma_{1}, \gamma_{2}\right) \in D_{1} \times\right.$ $\left.D_{2}\right\}$ where $D_{1}=\{1,1.1, \cdots, 1.4\}$ and $D_{2}=$ $\{1.5,1.6, \cdots, 1.9\}$. The i.i.d. Gaussian noise has covariance $\sigma^{2} I_{2}=I_{2}$.

In rate allocation, the quantizer is optimized using $\left(L_{d_{1}}^{b} \times L_{d_{2}}^{t}\right) \cup\left(L_{d_{2}}^{t} \times L_{d_{1}}^{b}\right)$. Fig. 3 shows the approximation of the optimal cost $\lambda=0.48$.

For comparison, an optimal dynamic quantization without rate allocation is also computed and the quantizer is optimized using $L_{d_{1}}^{b} \times L_{d_{2}}^{t}$. Hence the first sensor is always binary. The resulting optimal cost is $\tilde{\lambda}=0.483$. The static quantizer with $r=(0.4 ; 1,1.5)$ attains a cost 0.5 .

It is of interest to investigate (1) the advantage of dynamic rate allocation with a higher ratio between the number of states for $Z_{t}$ and that of the quantization levels, and (2) the selection of $L_{d_{1}}^{b}$ and $L_{d_{2}}^{t}$. This requires more computation and will be considered in future work.

\section{CONCLUSION}

This paper considers dynamic quantization and rate allocation in sensor networks by a stochastic control approach. Optimization of the network performance is achieved by feedback from the fusion center to the sensor nodes.

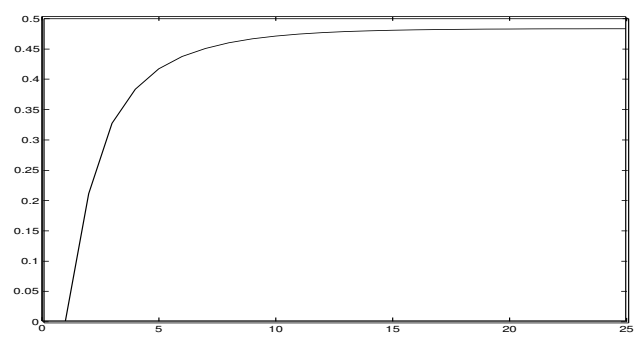

Fig. 3. The iterates converge to the optimal cost of the discretized Bellman equation. The step size for discretization is 0.02 .

\section{REFERENCES}

Alhakeem, S. and P.K. Varshney (1996). Decentralized Bayesian hypothesis testing with feedback. IEEE Trans. Syst., Man Cybern. 26, 503-513.

Bertsekas, D.P. (1995). Dynamic Programming and Optimal Control, Vol. 1,2. Athena Scientific. Belmont, MA.

Chamberland, J.-F. and V.V. Veeravalli (2003). Decentralized detection in sensor networks. IEEE Trans. Signal Process. 51, 407-416.

Chong, C.-Y. and S.P. Kumar (2003). Sensor networks: evolution, opportunuties, and challenges. Proc. IEEE 91, 1247-1256.

Fernandez-Gaucherand, E., A. Arapostathis and S.I. Marcus (1991). On the average cost optimality equation and the structure of optimal policies for partially observable Markov decision processes. Ann. Oper. Res. 29, 439-470.

Fletcher, A.K., S. Rangan and V. K. Goyal (2004). Estimation from lossy sensor data: jump linear modeling and Kalman filtering. In: Proc. Int. Symp. Inform. Process. Sensor Networks. Berkeley, CA. pp. 251-258.

Krishnamurthy, V. (2002). Algorithms for optimal scheduling and management of hidden Markov model sensors. 50, 1382-1397.

Kumar, P.R. and P. Varaiya (1986). Stochastic Systems: Estimation, Identification, and Adaptive Control. Pretice-Hall. Englewood Cliffs, NJ.

Mazor, E., A. Averbuch, Y. Bar-Shalom and J. Dayan (1998). Interacting multiple model methods in target tracking: a survey. IEEE Trans. Aerospace Electron. Syst. 34, 103-123.

Pados, D., K.W. Halford, D. Kazakos and P. Papantoni-Kazakos (1995). Distributed binary hypothesis testing with feedback. IEEE Trans. Syst., Man and Cybernetics 25, 21-42.

Shue, L., S. Dey, B.D.O. Anderson and F.D. Bruyne (2001). On state-estimation of a twostate hidden Markov model with quantization. IEEE Trans. Sig. Process. 49, 202-208. 